\title{
Europäische multiple Modernität als interzivilisatorische Konstellation
}

\section{Zur Transformation Europas durch europäische Integration und Erweiterung in einer sich globalisierenden Welt}

\section{Willfried Spohn}

Zusammenfassung: In der interdisziplinären und auch soziologischen Europaforschung dominieren modernisierungs- und eurozentrische Prämissen, das heißt die Erklärung des europäischen Mehrebenenregimes aus den transnationalen Spill-over-Prozessen sich modernisierender Gesellschaften in Europa. Im Kontrast wird hier ein an Shmuel Eisenstadt orientierter interzivilisatorischer, multipler Modernitätsansatz auf Europa und den europäischen Integrations- und Erweiterungsprozess umrissen. Für diesen Ansatz sind zentral: erstens die vielfältigen Modernen und Modernisierungsprozesse, vor allem auch im Blick auf die Transformationsprozesse im postkommunistischen Mittel- und Osteuropa; zweitens die internationalen und interzivilisatorischen Beziehungen der europäischen Gesellschaften zu anderen Zivilisationskomplexen; und drittens die globale Rolle Europas und die globale Beeinflussung Europas in einer sich globalisierenden Welt. Erst unter Berücksichtung dieses interzivilisatorischen und globalen Kontextes lassen sich die Dynamiken des europäischen Integrations- und Erweiterungsprozesses angemessen konzeptualisieren, analysieren und erklären.

Schlüsselwörter: Europäische multiple Moderne $\cdot$ Europäisierung $\cdot$ Europa als interzivilisatorische Konstellation · Globalisierung

\section{European multiple modernity as an interzivilisational constellation - The transformation of Europe through European integration and enlargement in a globalizing world}

\begin{abstract}
In interdisciplinary and sociological research on Europe, Euro-centric modernization premises are still dominant, i.e, the explanation of the European multi-level regime on the basis of transnational spill-over mechanisms originating from modernizing national societies in Europe. In contrast, this article will outline an inter-civilizational multiple modernities approach to Europe, based on the work of Shmuel Eisenstadt. Such an approach needs to focus on: 1. the multiplicity of modernization trajectories and modernities, particularly in view of the transformation processes
\end{abstract}

(C) VS-Verlag 2010

Dr. W. Spohn $(\triangle)$

Institut für Soziologie, Universität Göttingen, Platz der Göttinger Sieben 3,

37073 Göttingen, Deutschland

E-Mail: willfried.spohn@sowi.uni-goettingen.de 
in postcommunist Central and Eastern Europe; 2. the international and inter-civilizational relations and encounters between European and non-European civilizational complexes; and 3. the global role of Europe and the global impact on Europe in a globalizing world. Only in considering such an inter-civilizational and global context is it possible to adequately conceptualize, analyze and explain the dynamics of the European integration and enlargement process.

Keywords: European multiple modernity $\cdot$ Europeanization $\cdot$ Europe as inter-civilizational constellation · Globalization

\section{La modernité européenne multiple comme constellation intercivilisationnelle - Sur la transformation de l'Europe par l'intégration et l'élargissement européens dans un monde se mondialisant}

Résumé: La recherche interdisciplinaire mais aussi sociologique sur l'Europe est dominée par des prémisses modernisateurs et eurocentristes, c'est-à-dire par une explication du régime européen multi-niveau par les processus de spill over transnationaux de sociétés européennes se modernisant. Cet article appréhende au contraire l'Europe et le processus d'intégration et d'élargissement européens avec une approche de la modernité intercivilisationnelle et multiple inspirée de Shmuel Eisenstadt. Cette approche est axée sur les points suivants: premièrement, la multiplicité de la modernité et des processus de modernisation, notamment eu égard aux processus de transformation en Europe centrale et orientale post-communiste; deuxièmement, les relations internationales et intercivilisationnelles des sociétés européennes avec d'autres complexes civilisationnels; troisièmement, le rôle de l'Europe dans le monde et les influences s'exerçant sur l'Europe dans un monde se mondialisant. Les dynamiques du processus d'intégration et d'élargissement européens ne peuvent être convenablement conceptualisées, analysées et expliquées qu'en tenant compte de ce contexte intercivilisationnel et mondial.

Mots-clés: Modernité européenne multiple $\cdot$ Européisation · l'Europe comme constellation intercivilisationnelle $\cdot$ Mondialisation

\section{Einleitung}

Im Folgenden wird ein interzivilisatorischer multipler Modernitätsansatz zur europäischen Integration und Erweiterung im postkommunistischen Europa nach 1989 in einer globalen Perspektive skizziert. Unter dem Stichwort der europäischen multiplen Modernität beziehe ich mich namentlich auf den von Eisenstadt (2000, 2003) und seinen Mitarbeitern entwickelten zivilisationsvergleichenden Ansatz (Arnason et al. 2005). Dieser Ansatz kann am besten als kritische Revision der euro- und westzentrierten Modernisierungsund Globalisierungsansätze gesehen werden, die nach wie vor in den gegenwärtigen Sozial-, Politik- und Kulturwissenschaften dominieren. Anstatt das (west-)europäische und nordamerikanische Modell als universelles Modell der globalen Moderne zu verstehen, geht der Ansatz multipler Modernitäten von einer Vielzahl von Modernen und Modernisierungsprozessen im Kontext interzivilisatorischer und globaler Interaktionsbeziehungen und Strukturkonstellationen aus. In dieser Perspektive stellt wohl die (west-) europäische Entwicklung das originäre Modell von Moderne dar, doch ist es zugleich durch spezifische Charakteristika der europäischen Zivilisation geprägt, die es von den 
anderen zivilisatorischen Modernen unterscheiden und seine Entwicklungsdynamik im interzivilisatorischen und globalen Kontext bestimmen.

Ein solcher zivilisationsvergleichender und interzivilisatorischer multipler Modernitätsansatz ist für die Analyse Europas und dessen gegenwärtige Transformation durch den europäischen Integrations- und Erweiterungsprozess bisher nicht systematisch entwickelt worden. Auch die hier vorgelegte Skizze hat keinen derart systematischen Anspruch. Sie will aber vor dem Hintergrund der interdisziplinären und soziologischen Europaforschung (Bach 2000; Jachtenfuchs u. Kohler-Koch 1996; Loth u. Wessel 2001) und des dort vorherrschenden Eurozentrismus wenigstens in einigen Konturen die Richtung eines solchen multiplen Modernitätsansatzes zu Europa und zur Europäisierung angeben. So sehen die meisten interdisziplinären und soziologischen Ansätze in der Europaforschung den europäischen Integrations- und Erweiterungsprozess als ein auf der Grundlage der Interessen der Mitgliedsstaaten entstehendes intergouvernementales und supranationales Mehrebenensystem und damit als eine im Wesentlichen europainterne Entwicklungsdynamik. Sie abstrahieren dabei allerdings von den sich wandelnden internen und externen Konstellationsbeziehungen der europäischen Zivilisation im interzivilisatorischen und globalen Kontext. Dieser Gefahr eines Eurozentrismus, der gewissermaßen den methodologischen Nationalismus auf der europäischen Ebene reproduziert, will diese Skizze der europäischen multiplen Modernität als einer interzivilisatorischen Konstellation begegnen (vgl. Spohn 2006).

Dazu will ich im Folgenden zunächst einige Kernpunkte meines Ansatzes europäischer multipler Modernität erläutern und dann eine zivilisationsanalytische Perspektive auf den Wandel Europas umreißen. Auf dieser Grundlage skizziere ich im Anschluss die interzivilisatorischen Rahmenbedingungen des europäischen Integrations- und Erweiterungsprozesses und gehe schließlich auf das Wechselverhältnis von Europäisierung und Globalisierung ein.

\section{Europäische multiple Modernität, Identitäten und Grenzziehungen}

Das Konzept der europäischen multiplen Modernität, das ich hier zugrunde lege, folgt dem in der Soziologie zunehmend einflussreichen Ansatz multipler Modernitäten, wie er von Shmuel Eisenstadt und seinen Mitarbeitern entwickelt wurde (Eisenstadt 2000, 2003). In der dabei entwickelten globalen Vergleichsperspektive dient er auch dazu, die zivilisatorischen Besonderheiten Europas präziser zu fassen (Eisenstadt 1987, 2003). Diese multiple Modernitätsperspektive auf Europa setzt sich vor allem gegen die in den Sozial- und Politikwissenschaften nach wie vor dominanten Modernisierungstheorien ab, für die Europa, und hier insbesondere Westeuropa, den Inbegriff der Moderne darstellt und als solche das Modell für die im Zuge der Globalisierung weltweiten Modernisierungsprozesse vorgibt. Unter Modernisierung wird dabei in unterschiedlichen Akzentsetzungen ein Bündel soziokultureller Prozesse verstanden, das Prozesse der sozialen bzw. funktionalen Differenzierung und Individualisierung, der Staatsformierung, Nationsbildung und Demokratisierung, der kapitalistischen Entwicklung und Industrialisierung sowie der Säkularisierung und kulturellen Pluralisierung umfasst (Knöbl 2001). Dieses komplexe Bündel von Modernisierung und Modernität hat sich, so die Grundprämisse 
der Modernisierungstheorie, zunächst in Europa entwickelt, universalisiert sich aber durch friedliche oder gewaltsame Durchdringung der Welt zu einer globalen Moderne (Martinielli 2005).

In diesem theoretischen Kontext stellt der Ansatz multipler Modernitäten eine spezifische Kritik der euro- oder westzentrierten Modernisierungs- und Globalisierungstheorie dar. Er akzeptiert durchaus die multidimensionale modernisierungstheoretische Fassung von Modernität, kritisiert allerdings den ihr zugrunde liegenden strukturfunktionalistischen Evolutionismus und teleologischen Universalismus. Er betont stattdessen die historische Kontextualität, die sozialen Handlungsgrundlagen, die Kontingenz, Fragilität und Widersprüchlichkeit von Modernisierungsprozessen und Modernität (Eisenstadt 1996; Knöbl 2007). Er akzeptiert ebenso die globalisierenden Entwicklungstendenzen von Modernisierung, kritisiert aber ihre euro- oder westzentrierte Konzeptualisierung und nimmt stattdessen die Vielfalt von Traditionen und Modernen sowie ihre variierende Kombination in unterschiedlichen Modernisierungspfaden an. Diese zivilisationsspezifische Vielfalt von Modernen gründet dabei nicht nur auf unterschiedlichen politischen und ökonomischen Strukturen, sondern vor allem auch auf unterschiedlichen Kulturen und Institutionen, unterschiedlichen kulturellen und politischen Programmen von Modernität und dadurch beeinflussten Identitäten und Grenzziehungen. Von besonderer Bedeutung sind hierbei Achsenzeitzivilisationen, Weltreligionen und Weltimperien sowie ihre historischen Langzeitwirkungen auf unterschiedliche Formen von Modernisierung und Modernität. In diesem Kontext stellt auch Globalisierung nicht eine allein westlich angetriebene Form der Entwicklung globaler Modernität dar, sondern kristallisiert sich auf der Grundlage unterschiedlicher Formen der Moderne und interzivilisatorischer Vernetzungen in Gestalt von multiplen Globalisierungen und Globalität aus (Robertson 1992; Robertson u. White 2006).

Eine häufig vorgetragene Kritik an diesem zivilisationsvergleichenden multiplen Modernitätsansatz besagt, dass dieser analog der international vergleichenden Modernisierungsforschung nicht mehr nationalstaatlich verfasste Gesellschaften, sondern nunmehr Zivilisationen als voneinander unabhängige Großeinheiten unterstellt. Doch gegenüber einer solchen Kritik definiert der multiple Modernitätsansatz zum einen Zivilisationen als historisch sich wandelnde Zivilisationskomplexe mit je eigener Zivilisationsdichte, spezifischen Zentrums- und Peripheriestrukturen, sozio-kulturellen Makro- und Mikroebenen, multiplen kollektiven und individuellen Akteuren sowie komplexen Identitätsbildungen und Grenzziehungen. Zum andern betont er die sich wandelnden interzivilisatorischen Beziehungsstrukturen und -interaktionen in Form von Austausch, Kooperation und Konflikt im Kontext der sich entwickelnden Weltgeschichte (Arnason et al. 2005). In einer solchen Perspektive stellt weder die europäische Moderne eine nur intern angetriebene Entwicklungsdynamik noch Globalisierung eine nur westlich angetriebene Form der Globalisierung dar. Vielmehr entwickelt sich die europäische Moderne in einem weltgeschichtlichen ökumenischen Raum (Hodgson 1993); ebenso entwickelt sich die globale Moderne auf der Grundlage vielfältiger Modernen und interzivilisatorischer Vernetzungen und damit in Formen vielfältiger Globalisierungen und Globalität (vgl. Schwinn 2006).

In einer solchen zivilisationsvergleichenden Perspektive stellt Europa wohl die originäre Form der Moderne dar. Sie unterscheidet sich jedoch trotz ihrer weltweiten Verbreitung 
durch Kolonialismus und Imperialismus in der weltgeschichtlichen Phase europäischer Welthegemonie durch eine Reihe von spezifischen Eigentümlichkeiten von anderen zivilisatorischen Modernen. Dabei sollte, erstens, der strukturelle und kulturelle Pluralismus der europäischen Zivilisation hervorgehoben werden (Eisenstadt 1987; Rokkan 1975). Er besteht aus einer komplexen Matrix von politischen, ökonomischen und kulturellen Zentrums- und Peripheriestrukturen sowie vielfältigen kulturellen, religiösen und säkularen Mentalitätsgrundlagen und -strömungen. Zweitens entwickeln sich in diesem Rahmen mit der Moderne spezifische Machtgefälle zwischen Zentren und Peripherien. Sie bilden die Grundlage von mindestens vier (Davies 1996; Gellner 1983; Szücs 1991) historischen Regionen der Staats- und Nationsbildung in West-, Mittel-, Ostmittel- und Osteuropa. Drittens, quer zu diesen historischen Regionen, aber auch in Kombination mit ihnen, konstituieren sich durch die Entwicklung der religiösen Haupttypen von Katholizismus, Protestantismus, christlicher Orthodoxie und Islam spezifische Zonen von religiöser Entwicklung und Säkularisierungsmustern (Martin 1978, 2005; Spohn 2003) heraus. Viertens kristallisieren sich parallel zu diesen politischen Machtstrukturen und -gefällen kulturelle Machtgefälle, Superioritäts- und Inferioritätskomplexe heraus: Imperiale Zentren verstehen sich als Zivilisationsmodelle, die von den Peripherien übernommen oder ihnen oktroyiert werden (Barth u. Osterhammel 2005). Fünftens graben sich diese politischen und kulturellen Machtgefälle in die Formierung kollektiver Identitäten und Grenzziehungen ein. Dabei spielen sowohl externe Grenzziehungen zwischen Europa und den angrenzenden Zivilisationsräumen als auch interne Grenzziehungen zwischen den verschiedenen politischen und kulturellen Zonen in Europa eine zentrale Rolle.

Schließlich sollte die europäische Zivilisation mit ihrem strukturellen und kulturellen Pluralismus sowie ihren ökonomischen, politischen und kulturellen Zentrums- und Peripherierelationen nicht statisch gefasst werden - wozu der makrostrukturelle Ansatz von Stein Rokkan und Shmuel Eisenstadt verleiten könnte -, sondern als ein sich historisch wandelnder räumlicher, sozialer, kultureller und kognitiver Konstruktions- und Rekonstruktionsprozess (Delanty 1995). Auch sollte - stärker als es Eisenstadt in seiner zivilisationsvergleichenden Analyse Europas oder Rokkan in seiner analytischen Europakarte tun - der historisch sich wandelnde interzivilisatorische und globale Bedingungs- und Interaktionskontext in der Konstruktion und Rekonstruktion der europäischen Zivilisation betont werden (Osterhammel 2008). In einer solchen historisch-interzivilisatorischen Orientierung ist es in meinen Augen gerechtfertigt, nicht nur von der europäischen Moderne im Singular, sondern von den multiplen Modernitäten Europas im Plural zu reden und diese historisch sich wandelnde interzivilisatorische Konstellation auch als Grundlage für den nach dem Zweiten Weltkrieg unter spezifischen Bedingungen einsetzenden europäischen Integrationsprozess zu sehen.

\section{Eine historisch-interzivilisatorische Perspektive auf Europa}

Aus euro- bzw. westzentrischem Blick - in den Sozial-, Politik- und Kulturwissenschaften nach wie vor die dominante Sichtweise - ist die Entwicklung der europäischen Zivilisation und ihre weltweite Verbreitung das Resultat ihrer eigenen internen Entwicklungsdynamik: von den allgemeinen Grundlagen Europas in der griechischen, römischen und 
schließlich christlichen Zivilisation, seiner Formierung nach dem Zerfall des Römischen Reichs im Karolingischen Reich, der Entstehung des Feudalismus und der Ausbreitung des Christentums über den Durchbruch der europäischen Moderne in Renaissance, protestantischer Reformation, katholischer Gegenreformation und Reform sowie humanistischer Aufklärung bis zum Aufstieg des Westens in der politischen und industriellen Doppelrevolution und auf diesen Grundlagen die Entwicklung der Hegemonie Westeuropas und des Westens und schließlich in diesem Rahmen die zunehmende Verwestlichung der nicht-europäischen Welt durch sich universalisierende Prozesse der Modernisierung. Auch wenn der Aufstieg des Westens über lange Strecken mit Kolonialherrschaft und Imperialismus verbunden war und sich mit rassistischen und imperialistischen Kulturhierarchien verband, ist doch die progressive Verwestlichung der Welt letztlich, so die weitverbreitete modernisierungstheoretische Annahme, der Superiorität der westlichen Moderne gegenüber den traditionellen Gesellschaftsformen in den nichteuropäischen Weltregionen geschuldet.

Aus einer interzivilisatorischen Perspektive ist diese euro- und westzentrische Sichtweise allerdings zunehmend einer grundlegenden Kritik unterworfen worden. So hat die neue interzivilisatorische Weltgeschichte im Kern die ursprüngliche Abhängigkeit der Entstehung und Entwicklung Europas von den asiatischen Imperien und Zivilisationsräumen - oder in der überspitzten Formel John Hobsons (2004): ,die östlichen Ursprünge des Westens“" herausgearbeitet. Danach stellte Europa für gut Tausend Jahre einen weltregionalen Hinterhof dar und war vor allem durch die rasch expandierende islamische Zivilisation bedroht, die große Teile des zerfallenden Römischen Reiches eroberte und dabei viele ökonomische, rechtliche, kulturelle und wissenschaftliche Ressourcen der griechischen, römischen und jüdischen Zivilisationen übernahm und in eigener Form weiterentwickelte. Zugleich fungierte die islamische Zivilisation auch als Mittler zwischen den östlichen Zivilisationen Chinas und Indiens. Insbesondere China hatte für lange Zeit in weiten Bereichen eine ökonomische, technologische und wissenschaftliche Führungsstellung inne, hatte die meisten Elemente der industriell-kapitalistischen Entwicklung Europas schon deutlich früher entwickelt und war Europa in seinem ökonomischen Entwicklungsniveau bis an die Schwelle der industriellen Revolution überlegen (Pomeranz 2000). Auch Indien entwickelte vor der Eroberung durch Großbritannien ein beträchtliches ökonomisch-technologisches Niveau (Bayly 2008). Die islamische Zivilisation mit ihrem politischen Zentrum des Osmanischen Reiches fungierte als Mittler dieser östlichen Globalisierung. Entsprechend ist es angemessener, die über lange Zeit nur allmählich fortschreitende europäische Entwicklung als einen Lern- und Adaptionsprozess an die Zivilisationsdynamiken des Ostens zu fassen, bevor dann die europäische Zivilisationssynthese ihre besondere moderne Entwicklungsdynamik entfaltete.

Auf einer solchen interzivilisatorischen Perspektive basierte der um 1500 einsetzende Aufstieg Westeuropas und der damit beginnende europäische Kolonialismus vor allem auf einer globalen Umpolung der ökonomischen, technologischen und wissenschaftlichen Ressourcen der östlichen Zivilisationen im Verbund mit der Entstehung und Entwicklung der atlantischen Zivilisation auf der Grundlage von Kolonialherrschaft und Sklavenwirtschaft in dem durch Europa ,neu entdeckten“ Amerika (Smith 2006). Dieser globale geoökonomische Umpolungsprozess war einerseits begleitet von einem Aufstieg der westeuropäischen atlantischen Staaten - zunächst Portugal und Spanien, dann 
Holland, Großbritannien und Frankreich - zu imperialen Kolonialmächten, andererseits von einer Machtausweitung des mitteleuropäischen Deutschen und des Habsburger Reiches sowie des osteuropäischen russischen Zarenreiches. Beide Entwicklungen unterminierten langfristig die überkommene Machtbasis der östlichen Imperien. Trotz dieses einsetzenden geopolitischen Wandels bestand bis Mitte des 18. Jahrhunderts noch ein gewisses Machtgleichgewicht zwischen den westeuropäischen Kolonialmächten, den mittel- und osteuropäischen Imperien sowie den östlichen Imperien - allerdings im Verbund mit den kolonialen Machthierarchien im Atlantik und den sich verschärfenden Entwicklungsunterschieden zu Osteuropa. Entsprechend herrschte noch im 18. Jahrhundert eine europäische Haltung vor, die die zivilisatorische Überlegenheit oder Ebenbürtigkeit der asiatischen Zivilisationen implizit oder explizit anerkannte, aber zugleich die eigene zivilisatorische Überlegenheit gegenüber den „primitiven“ Gesellschaften Amerikas, Afrikas und Asiens voraussetzte. Diese europäische Haltung fand auch in der europäischen Aufklärung ihren Niederschlag, die - wie Jürgen Osterhammel in seiner Analyse der „Entzauberung Asiens“ (1998) nachweist - eher durch Respekt, Offenheit und Lernbegierde als durch kulturelle Superioritätsgefühle gekennzeichnet war. Zugleich verband sich diese europäische Haltung im Rahmen der verschiedenen Formen von Kolonialherrschaft jedoch mit unterschiedlichen christlichen und aufklärerischen Zivilisationsmissionen gegenüber den militärisch und ökonomisch unterlegenen Völkern Amerikas, Afrikas, Nordasiens und Ozeaniens (Barth u. Osterhammel 2005).

Erst mit der Entwicklung der europäischen Welthegemonie im Laufe des 19. und frühen 20. Jahrhunderts intensivierte sich nicht nur die koloniale Durchdringung nun auch der alten asiatischen Zivilisationen, sondern formierte sich auch eine kulturelle Welthierarchie, in der Europa zum universalen Modell der Moderne aufstieg. Die Grundlage war der sich dynamisch entfaltende Industriekapitalismus, zunächst in Großbritannien und Nordwesteuropa, dann aber auch im neu gegründeten Deutschen Reich, den USA mit ihren massiv anwachsenden Einwanderungsströmen und dem sich modernisierenden Japan. Diese dynamische weltwirtschaftliche Entwicklung verband sich mit einem parallelen Wachstum politisch-militärischer Machtpotenziale der führenden imperialen Nationalstaaten - sowohl der europäischen Großmächte als auch des aufsteigenden Japans (Mann 1993; Osterhammel 2008). Zugleich entstanden damit auch innerhalb Europas zunehmende ökonomische Entwicklungsgefälle zwischen Zentren und Peripherien, wachsende politische und militärische Ungleichgewichte und sich verschärfende Machtkämpfe zwischen den imperialen Nationen - Machtgefälle, die zugleich auf die kulturelle Geografie Europas zurückschlugen. Die westeuropäischen Länder, allen voran Frankreich und Großbritannien, verstanden sich als Speerspitze moderner Zivilisation mit weltweiten Zivilisationsmissionen. Das Deutsche und das Habsburger Reich definierten sich ebenfalls in universaler Mission als europäische Mitte mit einer Kulturmission gegen die bloß ,materielle“ westliche Zivilisation und die östliche ,rückständige Barbarei“. Die östlichen Imperien gerieten zunehmend ins Hintertreffen, orientierten sich am westlichen Modernisierungsmodell, behaupteten in Abwehr des Westens aber zugleich ihre eigene zivilisatorisch-spirituelle Überlegenheit: das zaristische Russland als Zentrum der christlichen Orthodoxie gegenüber dem sibirischen nördlichen Asien, das Osmanische Reich als Zentrum des Islam gegenüber dem turkmenischen Zentralasien und auch Japan gegenüber dem zerfallenden Chinesischen Reich. Im Rahmen dieser west-östlichen Kulturhierarchie 
waren die Konkurrenz- und Machtkämpfe zwischen den imperialen Nationen Europas, die schließlich mit dem Ersten und Zweiten Weltkrieg auch zum Verlust der europäischen Welthegemonie führten, deshalb auch nicht nur ökonomische, politische und militärische Auseinandersetzungen, sondern - wie Elias (1969) exemplarisch am Beispiel Deutschlands und Frankreichs zeigt - zugleich Kulturkämpfe zwischen universalistisch orientierten Zivilisationsmissionen.

Wie dynamisch und einflussreich auch immer diese Entwicklung der europäischen Welthegemonie war, kam diese doch ebenso zu einem plötzlichen und unerwarteten Ende. Zum einen führte der innereuropäische nationalistische und imperialistische Konkurrenzkampf zu zwei europäischen Weltkriegen, die die frühere europäische Vormachtstellung zerstörten. Dies betraf sowohl die ökonomische Zentrumsstellung, die nun an die Vereinigten Staaten und die sich industrialisierende Sowjetunion überging, als auch die politisch-militärische Suprematie, die durch die Teilung Europas seitens der zwei aufsteigenden Supermächte beendet wurde. Zum anderen transformierten sich die östlichen Imperien durch modernisierende Revolutionen, die ihre frühere Randstellung durchbrachen. Die Oktoberrevolution in Russland von 1917 führte zu einer ideokratischen Autokratie, die eine forcierte Industrialisierung ermöglichte und zugleich in der neuen Form der Sowjetunion die imperialen Grundlagen des zaristischen Reiches bewahrte. Die kemalistische Revolution transformierte das Zentrum des Osmanischen Reiches in einen modernen, europäisch orientierten säkularistisch-autoritären Nationalstaat. Die maoistische Revolution erneuerte das zerfallende chinesische Imperium und bereitete damit auch dessen ökonomischen Wiederaufstieg vor. Und nicht zuletzt ermöglichte der Niedergang der europäischen Welthegemonie auch die beschleunigte Dekolonialisierung der europäischen Kolonien in Asien, Afrika und Ozeanien. Im Ergebnis führte dieser Niedergang der früheren europäischen Welthegemonie auch zu einer Zerstörung der europäischen universalen Zivilisationsmission. So verlagerte sich diese nun auf die USA als neues westliches Weltzentrum; zugleich definierte sich Europa als eine - zudem bedrohte - Zivilisation unter den anderen Weltzivilisationen (Kaelble 2005).

\section{Europäische Integration, europäische Erweiterung und die interzivilisatorische Transformation Europas}

Der europäische Integrationsprozess, der nach dem Zweiten Weltkrieg unter den geopolitischen Bedingungen eines geteilten Europas in Westeuropa begann und sich nach dem Zusammenbruch des Sowjetkommunismus nach Ost- und Südosteuropa ausweitete, ist vor allem als Reaktion auf die Zerstörung Europas durch den Ersten und insbesondere den Zweiten Weltkrieg und mit dem Ziel der Überwindung ihrer Ursachen zu sehen. Entsprechend richteten sich die Integrationsimperative vor allem auf die Überwindung des Nationalismus durch den Aufbau einer europäischen Friedensordnung, die Lösung der deutschen Frage durch die Einbindung und Kontrolle Deutschlands, die Entwicklung einer sozial abgefederten kapitalistischen Marktwirtschaft und die Schaffung einer sozialen Wohlfahrtszone sowie den Aufbau und die Stabilisierung demokratisch-konstitutioneller Regime als Schutz vor autoritären und totalitären Abwegen. In der interdisziplinären und soziologischen Europaforschung wird dieser Integrations- und Erweiterungsprozess 
vornehmlich als innereuropäische, intergouvernementale und supranationale Entwicklungsdynamik analysiert (Bach 2000; Jachtenfuchs u. Kohler-Koch 1996; Loth u. Wessel 2001; dagegen Münch 2008; Vobruba 2001). Im Vordergrund stehen dabei die ökonomischen, politischen und rechtlichen Dimensionen dieses europäischen Integrationsprozesses, während die kulturellen Wandlungsprozesse, die mit diesen strukturellen Transformationsprozessen einhergehen, bisher nur am Rande eine Rolle gespielt haben (Viehoff u. Segers 1999; Wobbe 2005) und vornehmlich nur als deren sekundäre Folgewirkungen einbezogen worden sind. Doch findet der sich erweiternde europäische Integrationsprozess unter spezifischen geopolitischen Rahmenbedingungen und interzivilisatorischen Konstellationsveränderungen statt, ohne die seine interne Entwicklungsdynamik nicht zu erklären ist.

Für die Umsetzung der „europäischen Idee“ in eine transnationale Institutionenordnung war entscheidend, dass Europa nach dem Zweiten Weltkrieg geteilt wurde. Dadurch entstand in Westeuropa unter US-amerikanischem Schutzschild und in antikommunistischer Frontstellung gegen das nach Mitteleuropa vorgerückte kommunistische Sowjetimperium ein homogenerer und zugleich begrenzterer Raum im Vergleich zur Zeit nach dem Ersten Weltkrieg. Unter diesen spezifischen geopolitischen und interzivilisatorischen Rahmenbedingungen wurden zum einen die Rekonstruktion der westeuropäischen Nationalwirtschaften erleichtert und die Voraussetzungen für ihre allmähliche wirtschaftsinstitutionelle Vernetzung in Form der EWG, der Einbeziehung der EFTA und der Schaffung eines einheitlichen westeuropäischen Marktes geschaffen. Unter diesen Rahmenbedingungen wurden zum anderen die Transition von autoritären politischen Regimen und die Entwicklung und Konsolidierung von konstitutionellen Demokratien befördert sowie durch die entstehende politisch-rechtliche transnationale Institutionenordnung abgesichert. Diese ökonomische und politisch-rechtliche Integrationsdynamik basierte jedoch auch auf einer sich generalisierenden christlich-humanistischen Werteordnung, die sich gegen die totalitären Komponenten der europäischen Moderne durchsetzte und die Grundlage der kulturellen Integration Europas und der sich konturierenden europäischen Identität bildete (vgl. Judt 2005; Kaelble 2007; Therborn 1995).

Diese Transformation Westeuropas (Wallace 1994) in das mit Maastricht 1992 etablierte intergouvernemental-supranationale Mehrebenensystem der Europäischen Union war aber wiederum den enormen geopolitischen Wandlungsprozessen geschuldet, die sich mit dem Zusammenbruch des sowjetkommunistischen Systems und der Öffnung Osteuropas ergaben. Jetzt bestand die strukturelle und geopolitische Notwendigkeit, der europäischen Institutionenordnung eine feste supranationale Gestalt zu geben, sie durch eine gemeinsame Währung zu festigen und eine gemeinsame Sicherheitspolitik abzusichern sowie die supranationale Ebene durch eine sich vertiefende vertikale Integration zu stärken und gleichzeitig legislativ und parlamentarisch stärker zu kontrollieren. Diese vertikale Europäisierung betraf eine Vielzahl von Sektoren: von der Industrie- und Agrarpolitik über die Wirtschafts- und Sozialpolitik, die Bildungs- und Wissenschaftspolitik bis hin zur Rechts- und Kulturpolitik (Holzinger et al. 2008). Dadurch kam eine ökonomische Umverteilung durch Struktur- und Regionalfonds zwischen den Mitgliedsstaaten zustande, erfolgte eine Stabilisierung administrativer und konstitutioneller Strukturen, wurden kultureller und wissenschaftlicher Austausch befördert und vor allem die universellen Menschenrechte gegenüber sozialen, kulturellen und religiösen Diskriminierungen 
gestärkt. Diese mehrdimensionale Europäisierung kam vor allem den kleinen Nationalstaaten gegenüber den europäischen Großmächten zugute, da sie die Peripherien gegenüber den Machtzentren in Europa stärkte.

Das westeuropäische transnationale Mehrebenensystem, wie es sich in der Europäischen Union kristallisierte, war auch der Hauptmechanismus, die östliche Hälfte Europas nach dem Zusammenbruch der sowjetkommunistischen Herrschaftsordnung durch die Erweiterung nach Osten an sich zu binden und sukzessive zu integrieren (Schimmelfennig u. Sedelmeier 2005). Nach der gerade erlangten nationalen Unabhängigkeit gab es freilich bei allen neuen Beitrittskandidaten - in der ersten Runde acht ostmitteleuropäische und in der zweiten Runde zwei weitere südosteuropäische Länder - zunächst eine deutliche Ambivalenz, sich sofort wieder einem anderen transnationalen Regime anzuschließen und damit einen abhängigen und peripheren Status zu erneuern. In dieser Perspektive wurde die EU-Osterweiterung als neuer Versuch einer europäischen Imperiumsbildung oder auch Wiedererlangung eines germanisierten Europas gesehen (Boatcă u. Spohn 2010). Doch erfolgten die Beitritte 2004 und 2007 dann mit weitgehend großer Zustimmung - vor allem der Parlamente, weniger seitens der Bevölkerungen. Der Beitritt auf Grundlage der Kopenhagener Kriterien versprach sowohl eine forcierte ökonomische Entwicklung und eine Stabilisierung sowie Konsolidierung der postkommunistischen Demokratien als auch politischen Schutz vor einer erneuerten imperialen Abhängigkeit vom post-sowjetischen Russland. Mit dem vollzogenen Beitritt griffen nun die gleichen Transformationsmechanismen der vertikalen und sektoralen Integration wie in den westeuropäischen Mitgliedsländern vorher auch. Die Entwicklung marktkapitalistischer Wirtschaften war begleitet von der redistributiven Förderung peripherer Wirtschaftsbereiche durch Struktur-, Regional- und Agrarfonds, wenn auch auf einem deutlich niedrigeren Niveau als in der westeuropäischen Süderweiterung. Die politische Stabilisierung der postkommunistischen Demokratien wurde durch die Stärkung administrativer Strukturen und konstitutioneller Institutionen befördert. Hierbei spielte gerade der Abbau von ethnonationalen Spannungen sowohl zwischen den osteuropäischen Nationalstaaten als auch in ihnen durch die Förderung von ethnischen, kulturellen und religiösen Minderheitenrechten eine wichtige Rolle.

Aus einer multiplen Modernitätsperspektive stellt die Osterweiterung der Europäischen Union allerdings nicht einfach eine Verwestlichung der ostmittel- und südosteuropäischen Gesellschaften in Form einer nachholenden Modernisierung und Adaption an das westeuropäische institutionelle Mehrebenensystem dar. Vielmehr hat das unterschiedliche vorkommunistische wie kommunistische Erbe in den sozio-ökonomischen, politischrechtlichen wie auch kulturell-religiösen Dimensionen einen deutlichen Einfluss auf die Formen der postkommunistischen Transformations- und Entwicklungsprozesse ausgeübt. Dies zeigt sich, erstens, in den Formen eines peripheren Kapitalismus, in der Schwäche innerer konstitutionell-administrativer Staatsbildung sowie in der Stärke eines organischen, religiös geprägten Nationalismus - Komponenten, die alle einen Einfluss auf die spezifischen Strukturen von Demokratie und politischer Kultur haben (Linz u. Stepan 1996). Ebenso hat, zweitens, die Inkorporation einer großen Anzahl von postkommunistischen Ländern in die Europäische Union einen gewichtigen Einfluss auf die Struktur des europäischen Mehrebenensystems selbst gehabt. So spielt hier nicht nur die rein numerisch große Zunahme der Mitgliedsländer eine zentrale Rolle, die eine Restrukturierung 
des europäischen Mehrebenensystems - wie sie nun auch in dem allgemein ratifizierten Lissabonvertrag ermöglicht wurde - zur Folge hat, sondern auch die zunehmende politische und kulturelle Einflussnahme dieser neuen Mitgliedsländer, was zu neuen Machtbalancen innerhalb der erweiterten Union führt (Beichelt 2004). Hier sind auch, drittens, die interzivilisatorischen Verlagerungen auf der Grundlage der verschiedenen europäischen Religionen von Katholizismus, Protestantismus, Orthodoxie, Judentum und Islam von Bedeutung. So kann nicht übersehen werden, dass die Osterweiterung und die sich abzeichnende Südosterweiterung sich hauptsächlich auf postkommunistische Länder im Einflussgebiet des westlich-lateinischen Christentums konzentriert hat, während die Inkorporation von weiteren christlich-orthodoxen und mehr noch islamischen Ländern deutlich umstrittener ist. Aber umgekehrt kann auch gesagt werden, dass die Ambivalenz der orthodoxen und wenigen islamischen Länder hinsichtlich des Beitritts zu dem westeuropäisch dominierten lateinisch-christlichen und säkularistisch-humanistischen Klub deutlich höher ist als in den lateinisch-christlichen Ländern Ostmittel- und Südosteuropas (Byrnes u. Katzenstein 2006).

Zugleich sind diese sich rekonstruierenden intrazivilisatorischen Grenzziehungen mit ihren Auswirkungen auf die Prozesse der Ausweitung der Europäischen Union auf Ostmittel-, Südost- und Osteuropa eng verwoben mit den interzivilisatorischen Beziehungen zu der angrenzenden russisch-eurasischen Zivilisation einerseits und der islamischen Zivilisation mit ihrem säkularisierten türkischen Zentrum andererseits (Spohn 2009). Das postsowjetische Russland versteht sich, trotz der Verluste seiner osteuropäischen und zentralasiatischen Einflusssphäre, sowohl als Mitglied des Europäischen Hauses und damit Teil der europäischen Zivilisation als auch - mit seinen nordasiatischen imperialen Räumen - als euroasiatische Zivilisation. Entgegen den evolutionistischen Annahmen der Transformationsforschung folgt es keinem demokratisch-kapitalistischen, sondern einem spezifisch russischen, liberalisiert-autokratischen, staatskapitalistischen und orthodox nationalistischen Entwicklungsweg. Dieser verbindet sich gleichzeitig mit der Tendenz, das frühere formale sowjetische Imperium in Form eines informellen Imperiums zu rekonstruieren, und bildet insofern eine entscheidende Gegenmacht gegen eine weitere Ausdehnung der Osterweiterung der Europäischen Union. Demgegenüber gehört die Türkei als das frühere Zentrum des Osmanischen Reiches und eines der wichtigsten Kerngebiete der islamischen Zivilisation nicht zu Europa, obwohl sie als Nachfolger des Byzantinischen Reiches und mit ihrer imperialen Ausweitung nach Südosteuropa einen großen Einfluss auf die europäische Zivilisation hatte. Zugleich versteht sie sich als ein am europäischen Modell orientierter modern-säkularer Nationalstaat zunehmend als europäisch und strebt deshalb auch die Mitgliedschaft der Europäischen Gemeinschaft und nun der Europäischen Union an. Trotz des Status eines Beitrittskandidaten und der begonnenen Beitrittsverhandlungen erscheint dies eher als unwahrscheinlich, da die Türkei aus der Sicht der Mehrheit der Bevölkerungen der Mitgliedsstaaten als unvereinbar mit den christlich-humanistischen Grundwerten der Europäischen Union erscheint (Leggewie 2005; Zürcher u. van der Linden 2004). Als Gegenbewegung rekonstruiert nun die Türkei ihrerseits stärker ihr osmanisches Erbe und sieht sich zunehmend als Mittler zwischen dem Nahen und Mittleren Osten, Nordafrika und Europa.

Vor dem Hintergrund einer solchen Sicht auf Europa als eine historisch konstruierte und rekonstruierte interzivilisatorische Konstellation und der Transformation Europas 
durch europäische Integration und Erweiterung lässt sich die Entwicklung einer europäischen Identität nicht allein, wie es immer noch häufig geschieht, als ein Derivat der Europäisierung und des damit entstehenden europäisierten Interaktions- und Kommunikationsraumes fassen. Vielmehr entwickelt sich eine solche europäische Identität in der konflikthaften Politisierung der zuvor historisch herausgebildeten und gewandelten kulturellen und zivilisatorischen europäischen multiplen Identitäten (Checkel u. Katzenstein 2009). Mit Blick auf die interzivilisatorische Konstellation der europäischen multiplen Modernität (Delanty u. Rumford 2005; Delanty 2006; Persson u. Strath 2007; Rumford 2009) ist es allerdings angemessener, nicht nur von historisch vielfältigen europäischzivilisatorischen Identitäten, sondern auch von durch den europäischen Integrations- und Erweiterungsprozess politisierten multiplen europäischen Identitäten auszugehen. Sie kristallisieren sich im Interaktionsprozess zwischen Europäisierung und europäisch-zivilisatorischen Identitäten, verbinden sich mit nationalen, regionalen und lokalen Ebenen und reflektieren zugleich die interzivilisatorischen Beziehungskonstellationen und Grenzziehungen (Knöbl et al. 2008; Zielonka 2001).

\section{Globalisierung, Europäisierung und interzivilisatorischer Wandel}

Aus der umrissenen multiplen Modernitätsperspektive kann der europäische Integrations- und Erweiterungsprozess nicht einfach nur als eine innereuropäische Entwicklungsdynamik und Identitätsformierung betrachtet, sondern muss auch als abhängig von den externen geopolitischen und interzivilisatorischen Machtbalancen gesehen werden. Dieses geopolitische und interzivilisatorische Beziehungs- und Machtfeld ist im Rahmen der Weltgesellschaft seinerseits durch globale Kräftekonstellationen und in der gegenwärtigen globalen Ära durch sich intensivierende Prozesse der Globalisierung beeinflusst. Unter Globalisierung verstehe ich, namentlich Roland Robertson (1992; Robertson u. White 2006) folgend, die sich intensivierende materielle und kognitive Vernetzung und Verdichtung der Welt. Sie schließt sozioökonomische, politisch-rechtliche sowie kulturelle, kommunikative und kognitive Dimensionen und Komponenten ein. Diese vielschichtigen Globalisierungsprozesse überschreiten zunehmend die nationalstaatlich organisierten gesellschaftlichen Vernetzungsräume, verleihen den transnationalen Räumen gegenüber den nationalgesellschaftlichen Räumen ein zunehmendes Gewicht und verbinden sich mit einem intensivierenden globalen Bewusstsein bzw. einer wachsenden Globalität. Aus einer multiplen Modernitätsperspektive sollten diese mannigfachen Prozesse der Globalisierung und vielfältigen Formen der Globalität als „Glokalisierungen“ gesehen werden, das heißt als grundsätzlich verwoben mit Zivilisationskomplexen, nationalen Gesellschaften und lokalen Lebenswelten. Die paradoxen Folgewirkungen dieser mannigfachen Globalisierungsprozesse für interzivilisatorische Beziehungskonstellationen sind einerseits eine Verdichtung von interzivilisatorischem Kontakt, Austausch und Kooperation, aber andererseits auch eine Intensivierung von Konkurrenz und Konflikt zwischen verschiedenen Zivilisationskomplexen und die für sie konstitutiven Weltreligionen und kulturellen Identitäten.

Aus einer solchen globalen Sicht war Europa aufgrund seiner imperialen und kolonialen Welthegemonie in der Moderne einer der Hauptträger von Globalisierungsprozessen 
und stellt auch heute - trotz des Verlusts dieser globalen Hegemoniestellung - neben den USA, Russland, Indien, China und Japan nach wie vor einen der wichtigsten regionalen Träger und Räume der sich intensivierenden Globalisierung dar. Eine zentrale Rolle spielen hier der sich nach 1989 dynamisch entfaltende Europäisierungsprozess und dessen Erweiterung nach Osten. So trägt er durch die Herstellung eines einheitlichen europäischen Marktes, die politisch-legale Vereinheitlichung des europäischen Raumes, die transnationale soziale Mobilisierung sowie die transnationale kulturelle und kommunikative Verdichtung massiv zum Abbau der inneren nationalstaatlichen Schranken und Grenzziehungen in Europa, aber auch zur Überschreitung der Grenzen zwischen Europa und den umliegenden Zivilisationsräumen bei und stellt insofern einen integralen Bestandteil der gegenwärtigen Globalisierungsphase dar. Die wichtigsten Mechanismen dieser sich intensivierenden Globalisierungsprozesse sind die wachsenden ökonomischen Ströme von internationalen Handelsbewegungen, Finanzen und Investitionen, der Aufbau, die Ausweitung und Verankerung transnationaler politischer und rechtlicher Institutionen, die sich intensivierenden kulturellen Austausch- und Kommunikationsprozesse und schließlich die damit verbundenen transnationalen sozialen Vernetzungen in Form des Wachstums internationaler Eliten, internationaler und transnationaler Migrationsströme und des globalen Massentourismus (Held u. McGrew 2000). In allen diesen transnationalen und globalen Prozessen spielt der europäische Integrationsprozess innerhalb seiner nach Osten erweiterten Grenzen, aber auch darüber hinaus in die angrenzenden Zivilisationsräume hineinwirkend, eine zentrale Rolle. Entsprechend verstärken sich auch die Bemühungen, über die Grenzen des erweiternden Europäisierungsprozesses hinaus neue Assoziationsräume zu schaffen. Beispiele hierfür sind die Mittelmeerunion als Zusammenarbeit der südeuropäischen, nordafrikanischen und levantinischen Mittelmeerstaaten und die geplante osteuropäische Partnerschaftsunion mit der Einbeziehung von Belarus, der Ukraine, Moldawien und des Kaukasus.

Gleichzeitig tragen diese, durch den erweiterten europäischen Integrationsprozess beförderten sozioökonomischen, politisch-rechtlichen und kulturell-kommunikativen Globalisierungen nicht nur zu intensivierten Formen von Austausch, Kooperation und Homogenisierung bei, sondern verstärken auch umgekehrt Formen soziokultureller Heterogenisierung, politisch-militärischer Disparitäten und kulturell-religiöser Spannungen. So sind die wachsenden sozioökonomischen und politischen Globalisierungsprozesse mit einer Restrukturierung der europäischen und transeuropäischen Zentrums- und Peripherierelationen verbunden, und zwar in Form eines stärkeren Ausgleichs der internationalen Ungleichgewichte zwischen den europäischen Mitgliedsstaaten, einer parallelen Vertiefung der nationalgesellschaftlichen Disparitäten und Fragmentierungen zwischen hochentwickelten, rückständigen und ausgeschlossenen Sektoren sowie einer Zunahme der sozioökonomischen und politisch-militärischen Machtdifferenzen zwischen dem erweiterten Raum der Europäischen Union und den angrenzenden europäischen und nichteuropäischen Regionen. Zugleich sind diese durch Globalisierungsprozesse beförderten sozioökonomischen Disparitäten, politischen Machtdifferenzen und kulturell-kommunikativen Verdichtungen verbunden mit einer Intensivierung von zivilisatorischen, kulturellen und religiösen Spannungen und Konflikten. So gehen die Intensivierung von sozioökonomischem Austausch und die Verstärkung von politischer und rechtlicher Kooperation nicht nur mit einer Verdichtung kultureller und kommunikativer Begegnung 
und Verständigung einher, sondern befördern auch die Wahrnehmung kultureller Unterschiede, Gegensätze, Spannungen und Konflikte mit sich abgrenzenden und ausgrenzenden Selbstdefinitionen, Identitätskonstruktionen und Grenzziehungen. Diese negativen Konsequenzen der kulturellen Globalisierungsprozesse stellen auch zentrale Ursachen für die kulturell-konfliktive Dynamik der vollzogenen Osterweiterung, der darüber hinaus geplanten Südosterweiterung und der in Entstehung begriffenen osteuropäischen und mediterranen Assoziationsräume dar.

Es sind vor allem drei Ebenen, auf denen sich diese durch Globalisierungsprozesse verstärkten kulturell-kognitiven Spannungsfelder und Konfliktlagen auf den sich erweiternden europäischen Integrationsprozess auswirken. Auf der interzivilisatorischen Ebene verschärfen sich die kulturellen Gegensätze und zivilisatorischen Grenzziehungen zwischen den aneinander angrenzenden drei Zivilisationskomplexen des katholisch-protestantischen West- und Mitteleuropas, des christlich-orthodoxen Südost- und Osteuropas sowie des angrenzenden islamischen Zivilisationsraumes. Diese führen, erstens, zu interzivilisatorischen Rekonfigurationen, Abgrenzungen und Spannungen zwischen den Zentren dieser Zivilisationskomplexe wie der Europäischen Union, der postsowjetischen Russischen Föderation und einer sich repositionierenden Türkei sowie auch den peripheren Zonen zwischen ihnen. Diese Globalisierungsprozesse tragen, zweitens, auf einer internationalen und intrazivilisatorischen Ebene zur Formierung von sozial hierarchisierten und fragmentierten Gesellschaften als Grundlage für soziale und kulturelle Konfliktlagen zwischen global partizipierenden und global ausgeschlossenen Sektoren bei. Eine besondere Rolle spielen dabei die sich verstärkenden internationalen Migrations- und Immigrationsprozesse und die dadurch generierten Asymmetrien zwischen nationalstaatlichen Mehrheitsgesellschaften und Minderheitsethnien. Diese sind, drittens, auf einer globalen Ebene eng verknüpft mit der Rekonstruktion der Beziehungszusammenhänge zwischen Europa und den postkolonialen, durch Europa ehemals kolonialisierten Gesellschaften, in denen die aus postkolonialen Gesellschaften stammenden Migrationsethnien innerhalb Europas eine zentrale Rolle spielten (Costa 2005).

Um diesen desintegrativen und konfliktiven Kräften der Globalisierungsprozesse und ihrer Folgen für ein erweitertes Europa entgegenzuwirken, bedarf es nicht nur der Verstärkung ökonomischer und politischer Kooperation. Darüber hinaus wäre es notwendig, sozialen Fragmentierungen und kulturellen Asymmetrien entgegenzuwirken und kulturellen Austausch und gegenseitige Verständigung zu fördern, um die sozioökonomischen, politischen und kulturellen Bruchlinien, Spannungen und Konflikte zu überbrücken. Ein erster Eckstein der Gegensteuerung gegen die zunehmend konfliktive Dynamik eines erweiterten Europas würde einen verstärkten Dialog zwischen dem europäischen Säkularismus und den verschiedenen christlichen, jüdischen und islamischen Religionsformen als Scharnier eines interreligiösen und -kulturellen Dialogs einschließen, um so zum Abbau der interzivilisatorischen kulturellen Hierarchien, Stereotype und Xenophobien beizutragen. Ein zweiter Eckstein einer solchen Gegensteuerung würde den interzivilisatorischen Dialog in Europa und seinen Nationalgesellschaften zwischen Mehrheitsgesellschaften und ethnisch-religiösen Minderheiten fördern. Ein solcher Dialog müsste durch Formen von kultureller Staatsbürgerschaft und religiösen Minderheitsrechten abgesichert sein, um die gegenwärtigen Strukturen religiös-kultureller Fragmentierung durch eine Stärkung kultureller und religiöser Pluralisierung zu überwinden. Ein dritter 
Eckstein hätte einen solchen interreligiösen und interzivilisatorischen Dialog durch eine intensivere Bekämpfung der durch ökonomische Globalisierungsprozesse verstärkten Formen sozialer Hierarchisierung, Fragmentierung und Exklusion zu untermauern - im Hinblick sowohl auf die nach wie vor großen sozioökonomischen Unterschiede innerhalb der erweiterten Europäischen Union als auch das wachsende sozioökonomische Entwicklungsgefälle zwischen ihr und den angrenzenden nichteuropäischen Regionen und Zivilisationen.

Im Ergebnis heißt dies, dass die Prozesse der Europäisierung und Globalisierung einerseits partiell synchron verlaufen und dadurch die überkommenen traditionellen ökonomischen, politischen und kulturellen Machtasymmetrien abschleifen - Globalisierung überbrückt die nationalen und zivilisatorischen Grenzen und Grenzziehungen und Europäisierung beschleunigt diese internationale und interzivilisatorische Überbrückung durch transnationale sozioökonomische, politisch-rechtliche und kulturelle Integrationsprozesse. Andererseits laufen die Prozesse der Europäisierung und Globalisierung partiell auch auseinander - Globalisierung geht mit wachsenden sozioökonomischen und politischen Machtdifferenzen einher und motiviert dadurch zugleich zivilisatorische, kulturelle und religiöse Divergenzen und Asymmetrien, und Europäisierung versucht zumindest innerhalb der Grenzen eines erweiterten Europas, diesen sozioökonomischen und politischen Zentrums- und Peripheriebeziehungen und kulturellen Asymmetrien entgegenzuwirken. In diesem Kontext ist die vollzogene Osterweiterung und eine weitergeführte Südosterweiterung der Europäischen Union mit einer Konfliktdynamik zwischen homogenisierenden und heterogenisierenden Kräften konfrontiert, die sich sowohl innerhalb der Europäischen Union zwischen den alten und neuen Mitgliedsländern als auch außerhalb von ihr zwischen der Europäischen Union in ihrer Beziehung zu den angrenzenden Zivilisationsregionen auswirkt.

\section{Fazit}

Aus einer modernisierungstheoretischen Perspektive hat Samuel Huntington in seiner These vom Zusammenprall der Kulturen die wachsende konfliktive Mobilisierung von Zivilisationsgrenzen in einer sich gegenwärtig verstärkt globalisierenden Welt diagnostiziert, wenn auch, wie viele Kritiker angemerkt haben, mit der Tendenz, Zivilisationen als einheitliche Kulturblöcke zu essenzialisieren. Aus einer multiplen Modernitätsperpektive, wie sie hier im Anschluss an Shmuel Eisenstadt und Gerard Delanty für Europa umrissen wurde, sollten Zivilisationen und damit auch Europa als zivilisatorische Komplexe und interzivilisatorische Konstellationen gesehen werden, die durch interne Spannungen, Widersprüche und Antinomien charakterisiert sind und als solche kooperative als auch konfliktive Beziehungen einschließen. In paralleler Weise neigen Globalisierungs- und Weltgesellschaftstheorien, die modernisierungstheoretisch die Emergenz einer einheitlichen globalen Moderne unterstellen, dazu, die intra- und interzivilisatorischen Differenzen, Divergenzen, Konflikte und Widersprüche zu unterschätzen. Daneben stehen interzivilisatorisch argumentierende Globalisierungstheorien wie die von Roland Robertson, die ein besonderes Gewicht auf die kulturellen und kognitiven Dimensionen von Globalisierung und Globalität legen und damit auch das Spannungs- und Konfliktpotenzial 
von kulturellen Globalisierungsprozessen erkennen. Doch auch hier bedarf es einer stärkeren Berücksichtigung der sozioökonomischen und politischen Machthierarchien, wie sie etwa von neomarxistischen Weltsystemansätzen betont werden, sowie der zivilisatorischen, kulturellen und kognitiven Superioritäts- und Inferioritätskomplexe, wie sie insbesondere von postkolonialen Ansätzen (Conrad u. Randeria 2002; Boatcă u. Spohn 2010) herausgearbeitet werden.

Ausgehend von der hier umrissenen interzivilisatorischen multiplen Modernitätsperspektive kann ein kosmopolitisches Europa nicht nur kritisch-allgemein als postnationale Konstellation (Habermas 1998) oder unter den globalen Bedingungen der Weltrisikogesellschaft (Beck u. Grande 2004) aufgefasst werden. Vielmehr muss es als spezifische interzivilisatorische Konstellation und deren Transformation durch das europäische Mehrebenensystem in einer sich globalisierenden Welt analysiert und auf diesen Grundlagen in einer multizivilisatorischen Perspektive kritisch reflektiert werden. Im Rahmen der interzivilisatorischen Konstellation Europas bedarf es dazu auch nicht eines eurozentrierten, sondern eines, in den Worten Michael Walzers, ,reiterativen“ Universalismus. Aus einer solchen multiperspektivischen kosmopolitischen Perspektive muss sich ein erweitertes Europa vor allem konzentrieren auf: (i) die zivilisatorischen, kulturellen und religiösen Asymmetrien innerhalb und außerhalb der Europäischen Union; (ii) die Gegensteuerung gegen diese Asymmetrien durch die Beförderung multikultureller und multireligiöser Formen von Staats- und Unionsbürgerrechten; (iii) die Abschleifung von sozioökonomischen und politischen Zentrums- und Peripherie-Relationen innerhalb der Europäischen Union sowie zwischen ihr und den angrenzenden europäischen wie nichteuropäischen Regionen; und (iv) die dialogisch-interzivilisatorische Überwindung der überkommenen kulturellen und kognitiven Superioritäts- und Inferioritätsstrukturen. Diese stellen in vielem ein Erbe der Imperienbildung in Europa und der europäischen Kolonialherrschaft in den nichteuropäischen Weltregionen dar, die sich nach wie vor in asymmetrischen Beziehungen innerhalb Europas sowie zwischen Europa und den nichteuropäischen Zivilisationen reproduzieren.

\section{Literatur}

Arnason, J., Eisenstadt, S., \& Wittrock, B. (Hrsg.) (2005). Axial civilizations and world history. Leiden: Brill.

Bach, M. (Hrsg.) (2000). Die Europäisierung nationaler Gesellschaften. Sonderheft der Kölner Zeitschrift für Soziologie und Sozialpsychologie 40. Wiesbaden: Opladen.

Barth, B., \& Osterhammel, J. (2005). Zivilisierungsmissionen. Konstanz: UVK.

Bayly, C. (2008). Die Geburt der modernen Welt. Frankfurt a.M.: Campus.

Beck, U., \& Grande, E. (2004). Das Kosmopolitische Europa. Frankfurt a.M.: Suhrkamp.

Beichelt, T. (2004). Die Europäische Union nach der Osterweiterung. Opladen: Leske + Budrich.

Boatcă, M., \& Spohn, W. (2010). Globale, multiple und postkoloniale Modernen. Theoretische und historisch-soziologische Perspektiven. München: Hampp (i.E.).

Byrnes, T., \& Katzenstein, P. (Hrsg.) (2006). Religion in an expanding Europe. Cambridge: Cambridge University Press.

Checkel, J., \& Katzenstein, P. (Hrsg.) (2009). European identity. Cambridge, Mass.: Cambridge University Press. 
Conrad, S., \& Randeria, S. (2002). Jenseits des Eurozentrismus. Frankfurt a.M.: Campus.

Costa, S. (2005). Vom Nordatlantik zum „Black Atlantic“. Postkoloniale Konfigurationen und Paradoxien transnationaler Politik. Bielefeld: transcript.

Davies, N. (1996). Europe - a history. Oxford: Oxford University Press.

Delanty, G. (1995). The invention of Europe. London: Routledge.

Delanty, G. (Hrsg.) (2006). Europe and Asia beyond east and west. Towards a new cosmopolitanism. London: Routledge.

Delanty, G., \& Rumford, C. (Hrsg.) (2005). Rethinking Europe - social theory and the implications of Europeanization. London: Routledge.

Eisenstadt, S. (1987). European civilization in comparative perspective. Oslo: Scandinavian University Press.

Eisenstadt, S. (1996). Antinomien der Moderne. Frankfurt a.M.: Surhkamp.

Eisenstadt, S. (2000). Vielfalt der Moderne. Weilerswist: Velbrück.

Eisenstadt, S. (2003). Comparative civilizations and multiple modernities. Leiden: Brill.

Elias, N. (1969). Der Prozess der Zivilisation. Frankfurt a.M.: Suhrkamp.

Gellner, E. (1983). Nations and nationalism. London: Verso.

Habermas, J. (1998). Die postnationale Konstellation. Frankfurt a.M.: Suhrkamp.

Held, D., \& McGrew, A. (Hrsg.) (2000). The global transformation reader. Oxford: Polity.

Hobson, J. (2004). The eastern origins of the west. Cambridge: Cambridge University Press.

Hodgson, M. (1993). Rethinking world history. Chicago: Chicago University Press.

Holzinger, K. et al. (2008). Die Europäische Union: Theorien und Analysekonzepte. Paderborn: Schöningh.

Jachtenfuchs, M., \& Kohler-Koch, B. (Hrsg.) (1996). Europäische Integration. Opladen: Leske + Budrich.

Judt, T. (2005). Postwar. A history of Europe since 1945. New York: Penguin.

Kaelble, H. (2005). European self-definition in the twentieth century. In K. Eder \& W. Spohn (Hrsg.), Collective memory and European identity. Effects on integration and enlargement (S. 17-35). Aldershot: Ashgate.

Kaelble, H. (2007). Sozialgeschichte Europas. München: Beck.

Knöbl, W. (2001). Spielräume der Modernisierung. Weilerswist: Velbrück.

Knöbl, W. (2007). Die Kontingenz der Moderne. Frankfurt a.M.: Campus.

Knöbl, W., Koenig, M., \& Spohn, W. (2008). Europäisierung, multiple Modernitäten und kollektive Identitäten - Religion, Nation und Ethnizität in einem erweiterten Europa. Forschungsprojekt. Hannover: VolkswagenStiftung.

Leggewie, C. (2005). Die Türkei und Europa. Frankfurt a.M.: Suhrkamp.

Linz, J., \& Stepan, A. (1996). Problems of transition and consolidation of democracy. Southern Europe, Latin America and post-communist eastern Europe. Baltimore, MD: Johns Hopkins University Press.

Loth, W., \& Wessel, W. (2001). Theorien europäischer Integration. München: Oldenbourg.

Mann, M. (1993). Sources of social power. Vol. 2. Cambridge, Mass.: Cambridge University Press.

Martin, D. (1978). A general theory of secularization. Oxford: Oxford University Press.

Martin, D. (2005). Secularization - towards a general theory. Aldershot: Ashgate.

Martinelli, A. (2005). Global modernization. Rethinking the project of modernity. Leiden: Brill.

Münch, R. (2008). Die Konstruktion Europas. Frankfurt a.M.: Campus.

Osterhammel, J. (1998). Die Entzauberung Asiens. Europa und die asiatischen Reiche im 19. Jahrhundert. München: Beck.

Osterhammel, J. (2008). Verwandlung der Welt. Eine Geschichte des 19. Jahrhunderts. München: Beck.

Persson, H.-A., \& Strath, B. (Hrsg.) (2007). Reflections on Europe. Defining a political order in time and space. Bern: Lange. 
Pomeranz, K. (2000). The great divergence. Europe, China and the modern world economy. Princeton: Princeton University Press.

Robertson, R. (1992). Globalization. Social theory and global culture. London: Routledge.

Robertson, R., \& White, K. (2006). Globalization. London: Sage.

Rokkan, S. (1975). Dimensions of state formation and nation building: A possible paradigm for research on variations in Europe. In C. Tilly (Hrsg.), The formation of national states in Europe (S. 562-600). Princeton: Princeton University Press.

Rumford, C. (Hrsg.) (2009). The Sage handbook for European studies. London: Sage.

Schimmelfennig, F., \& Sedelmaier, U. (Hrsg.) (2005). The Europeanization of central and eastern Europe. Ithaca, NY: Cornell University Press.

Schwinn, T. (Hrsg.) (2006). Vielfalt und Einheit der Moderne: kultur- und strukturvergleichende Analysen. Wiesbaden: VS Verlag für Sozialwissenschaften.

Smith, J. (2006). Europe and the Americas: State formation, capitalism and civilizations in Atlantic modernity. Leiden: Brill.

Spohn, W. (2003). Nationalismus und Religion. Ein historisch-soziologischer Vergleich West- und Osteuropas. In M. Minkenberg \& U. Willems (Hrsg.), Politik und Religion (S. 323-345). Politische Vierteljahresschrift, Sonderheft 33. Wiesbaden: Westdeutscher Verlag.

Spohn, W. (2006). Interdisziplinäre Europastudien - Die Perspektive multipler Modernitäten. In T. Beichelt, B. Chołuj, G. Rowe \& H.-J. Wagener (Hrsg.), Europäische Studien - Eine Einführung (S. 435-451). Wiesbaden: VS Verlag für Sozialwissenschaften.

Spohn, W. (2009). Europeanization, religion and collective identities in an enlarging Europe. European Journal of Social Theory, 12, 358-374.

Szücs, J. (1991). Die drei historischen Regionen Europas. Frankfurt a.M.: Kritik.

Therborn, G. (1995). European modernity and beyond. Trajectories of European societies, 19452000. London: Sage.

Viehoff, R., \& Segers, R. T. (Hrsg.) (1999). Kultur, Identität, Europa: über die Schwierigkeiten und Möglichkeiten einer Konstruktion. Frankfurt a.M.: Suhrkamp.

Vobruba, G. (2001). Integration und Erweiterung. Europa im Globalisierungsdilemma. Wien: Passagen Verlag.

Wallace, W. (1994). The transformation of western Europe. London: Royal Institute.

Wobbe, T. (2005). Die Verortung Europas in der Weltgesellschaft. Historische Europasemantik und Identitätspolitik der Europäischen Union. In B. Heintz, R. Münch \& H. Tyrell (Hrsg.), Weltgesellschaft. Sonderband 1 der Zeitschrift für Soziologie (S. 348-373). Stuttgart: Lucius \& Lucius.

Zielonka, J. (Hrsg.) (2001). Europe unbound. Enlarging and reshaping the boundaries of the EU. London: Routledge.

Zürcher, E., \& van der Linden, H. (2004). The European Union, Turkey and Islam. Amsterdam: Amsterdam University Press.

Willfried Spohn, Dr., Privatdozent am Institut für Soziologie der Freien Universität Berlin, z.Z. Forschungsleiter/-koordinator des Volkswagen Forschungsprojekts „Europäisierung, multiple Modernitäten und kollektive Identitäten - Religion, Nation und Ethnizität in einem erweiterten Europa“ an der Universität Göttingen. Forschungsschwerpunkte: historisch-vergleichende Soziologie, multiple Modernitäten und Globalisierung mit einer Spezialisierung auf Politik und Religion in Europa in zivilisatorisch-vergleichender Perspektive. Ausgewählte Veröffentlichungen: Politik und Religion in einer sich globalisierenden Welt, 2008; (mit A. Ichijo, Hrsg.) Entangled identities: Europe and the nation, 2005; (mit K. Eder, Hrsg.) Collective memory and European identity. Effects of integration and enlargement, 2005. 